\title{
Using the INSPIRAL program to search for gravitational waves from low-mass binary inspiral
}

\author{
Duncan A Brown (for the LIGO Scientific Collaboration) \\ University of Wisconsin-Milwaukee, Milwaukee, WI 53211, USA \\ and \\ California Institute of Technology, Pasadena, CA 91125, USA
}

Received 1 April 2005, in final form 1 July 2005

Published 6 September 2005

Online at stacks.iop.org/CQG/22/S1097

\begin{abstract}
The INSPIRAL program is the LIGO Scientific Collaboration's computational engine for the search for gravitational waves from binary neutron stars and sub-solar mass black holes. We describe how this program, which makes use of the FINDCHIRP algorithm, is integrated into a sophisticated data analysis pipeline that was used in the search for low-mass binary inspirals in data taken during the second LIGO science run.
\end{abstract}

PACS numbers: $07.05 . \mathrm{Kf}, 04.80 . \mathrm{Nn}$

\section{Introduction}

The search for gravitational waves that arise from coalescing compact binary systems-binary neutron stars and black holes-is one of the main efforts in LIGO data analysis conducted by the LIGO Scientific Collaboration (LSC). For the first science run of LIGO, we focused attention on the search for low-mass binary systems (binary neutron stars and sub-solar mass binary black hole systems) since these systems have well-understood waveforms [1]. For the second science run of LIGO, S2, we have extended our analysis to include searches for binary black hole systems with higher masses. This work will be described elsewhere [2]. Here we describe the refinements to the original S1 search for low-mass binary inspiral waveforms: binary neutron star systems [3] and sub-solar-mass binary black hole systems which may be components of the Milky Way Halo (and thus form part of a hypothetical population of Macroscopic Astrophysical Compact Halo Objects known as MACHOs) [4, 5]. In this paper, we focus on how the central computational search engine - the INSPIRAL program-is integrated into a sophisticated new data analysis pipeline that was used in the S2 low-mass binary inspiral search.

The INSPIRAL program makes use of the LSC inspiral search algorithm FINDCHIRP, which is described in detail in a companion paper [6]. This companion paper describes the algorithm that we use to generate inspiral triggers given an inspiral template and a single data segment. 
There is more to searching for gravitational waves from binary inspiral than trigger generation, however. To perform a search for a given class of sources in a large quantity of interferometer data we construct a detection pipeline.

The detection pipeline is divided into blocks that perform specific tasks. These tasks are implemented as individual programs. The pipeline itself is then implemented as a logical graph, called a directed acyclic graph or DAG, describing the workflow (the order that the programs must be called to perform the pipeline activities) which can then be submitted to a batch processing system on a computer cluster. We use the Condor high throughput computing system [7] to manage the DAG and to control the execution of the programs.

The structure of this paper will follow these various tasks (programs). In section 2 we provide an overview of these tasks and then describe each task in turn. In section 3 we provide a simple illustration of how these elements are combined into a workflow pipeline.

\section{Pipeline overview}

In LIGO's second science run S2 we performed a triggered search for low-mass binary inspirals: since we require that a trigger occur simultaneously and consistently in at least two detectors located at different sites in order for it to be considered as a detection candidate, we save computational effort by analysing data from the Livingston interferometer first and then performing follow-up analyses of Hanford data only for the specific triggers found. We describe the tasks and their order of execution in this triggered search as our detection pipeline.

An overview of the tasks required in the detection pipeline is as follows: (i) data selection with the DATAFIND program, as described in section 2.1, (ii) template bank generation with the TMPLTBANK program, as described in section 2.2, (iii) binary inspiral trigger generation (the actual filtering of the data) with the INSPIRAL program, as described in section 2.3, (iv) creation of a follow-up (in other detectors) template bank based on these triggers generated with the TRIGBANK program, as described in section 2.4, and finally (v) coincidence analysis of the triggers with the inspiral coincidence analysis program INCA, as described in section 2.5.

Figure 1 shows our workflow in terms of these basic tasks. Epochs of simultaneous Livingston-Hanford operation are processed differently depending on which interferometer combination is operating. Thus, there are several different sets of data: $\mathrm{L} 1 \cap(\mathrm{H} 1 \cup \mathrm{H} 2)$ is when the Livingston interferometer L1 is operating simultaneously with either the $4 \mathrm{~km}$ Hanford interferometer $\mathrm{H} 1$ or the $2 \mathrm{~km}$ Hanford interferometer $\mathrm{H} 2$ (or both) - these are all the data analysed by the S2 low-mass inspiral analysis-while $\mathrm{L} 1 \cap \mathrm{H} 1$ is when $\mathrm{L} 1$ and $\mathrm{H} 1$ are both operating, $\mathrm{L} 1 \cap(\mathrm{H} 2-\mathrm{H} 1)$ is when $\mathrm{L} 1$ and $\mathrm{H} 2$ but not $\mathrm{H} 1$ are operating, and $\mathrm{L} 1 \cap \mathrm{H} 1 \cap \mathrm{H} 2$ is when all three interferometers are operating. A full $\mathrm{L} 1$ template bank is generated for the $\mathrm{L} 1 \cap(\mathrm{H} 1 \cup \mathrm{H} 2)$ data and the $\mathrm{L} 1$ data are filtered with INSPIRAL. Triggers resulting from this are then used to produce triggered banks for follow-up filtering of $\mathrm{H} 1$ and/or $\mathrm{H} 2$ data. However, if both $\mathrm{H} 1$ and $\mathrm{H} 2$ are operating then filtering of $\mathrm{H} 2$ is suspended until coincident $\mathrm{L} 1 / \mathrm{H} 1$ triggers are identified by INCA. After a final coincidence check, final triggers are checked to see if they could be vetoed by association with detector misbehaviour, which, for the S2 inspiral analysis, was diagnosed by examining an auxiliary interferometer channel of the L1 analysis for glitches [3].

This pipeline is equivalent to simply filtering the data from the three interferometers and looking for coincident triggers. The triggered search is more computationally efficient because $\mathrm{H} 1$ and $\mathrm{H} 2$ data are filtered only if they could possibly produce a coincident trigger, i.e., only for times when there are L1 triggers produced. Furthermore, the bank of templates that needs to be analysed in follow-up searches of the $\mathrm{H} 1$ and $\mathrm{H} 2$ data is far smaller than if these data sets had been analysed seperately using a full template bank. 


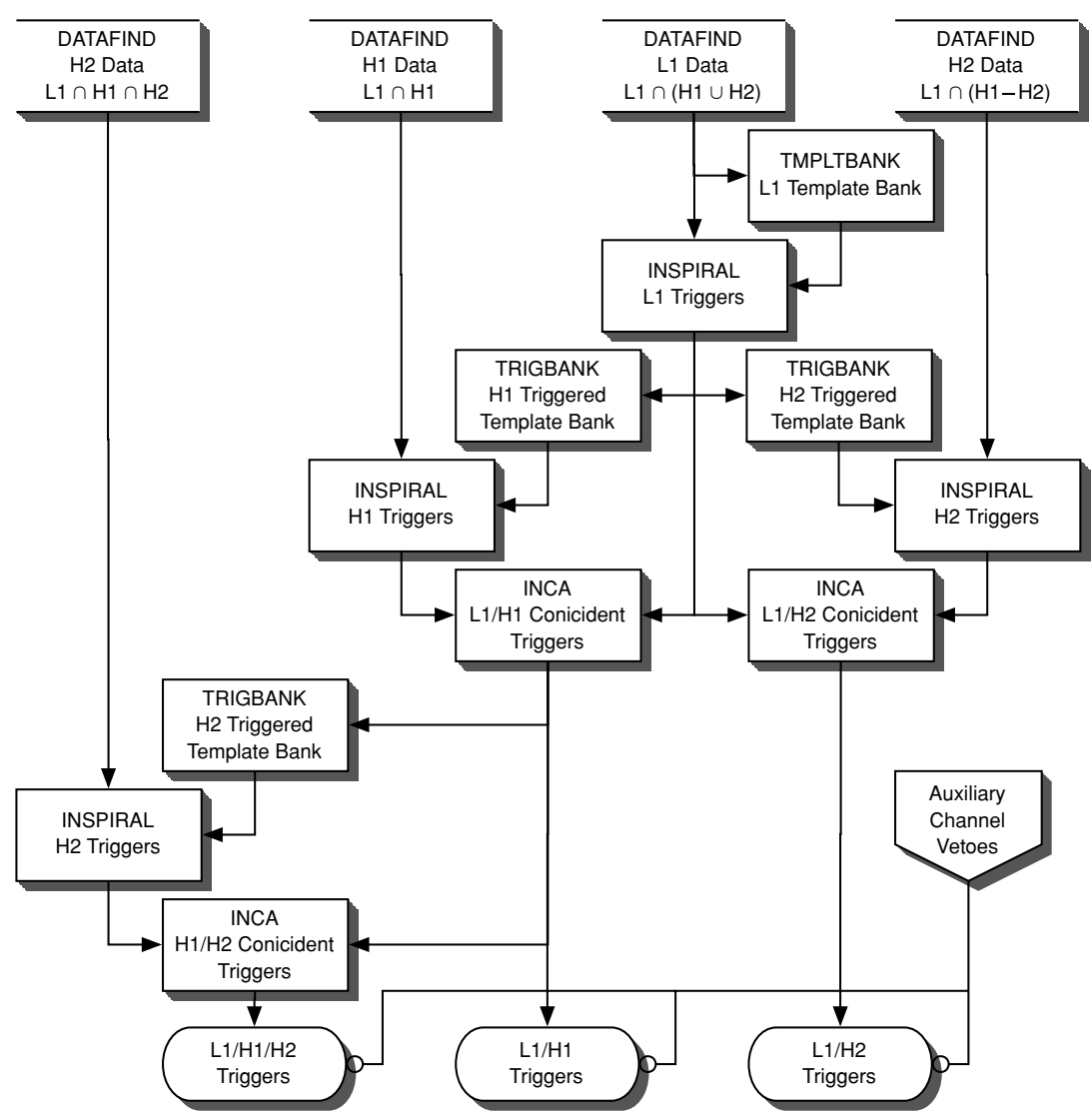

Figure 1. The triggered search pipeline. $\mathrm{L} 1 \cap(\mathrm{H} 1 \cup \mathrm{H} 2)$ indicates times when the $\mathrm{L} 1$ interferometer was operating in coincidence with one or both of the Hanford interferometers. $\mathrm{L} 1 \cap \mathrm{H} 1$ indicates times when the L1 interferometer was operating in coincidence with the H1 interferometer. $\mathrm{L} 1 \cap(\mathrm{H} 2-\mathrm{H} 1)$ indicates times when the L1 interferometer was operating in coincidence with only the $\mathrm{H} 2$ interferometer. The outputs of the search pipeline are triggers that belong to one of the two double coincident data sets or to the triple coincident data set.

Quantitatively, the S2 binary black hole MACHO search required an initial pass searching all the L1 data using the full L1 template bank. This initial search took $49.0 \mathrm{~h}$ on $4202.66 \mathrm{GHz}$ Pentium 4 Xeon CPUs to perform the matched filter and $\chi^{2}$ veto on the $406.8 \mathrm{~h}$ of L1 data with 14179 templates (on average) in the bank; in all, $126757744 \mathrm{~L} 1$ templates were filtered. The follow-up searches of $\mathrm{H} 1$ and $\mathrm{H} 2$ data required only $7.1 \mathrm{~h}$ and $1.1 \mathrm{~h}$ respectively, and the triggered-template banks in $\mathrm{H} 1$ and $\mathrm{H} 2$ were considerably smaller than the full bank in L1 was: the $\mathrm{H} 1$ bank contained an average of 2252 templates, the $\mathrm{H} 2$ bank triggered from $\mathrm{L} 1$ contained an average of 2665 templates and the $\mathrm{H} 2$ bank triggered from coincidence in L1 and $\mathrm{H} 1$ contained only 5 templates in total. The total numbers of templates filtered were 2213392 for $\mathrm{H} 1$ and 367800 for $\mathrm{H} 2$. The triggered search reduces the required computational resources considerably: had we performed a flat search, in which all the $\mathrm{H} 1$ and $\mathrm{H} 2$ data were filtered with the full $\mathrm{H} 1$ and $\mathrm{H} 2$ template banks, we estimate that the computation of the matched filter and $\chi^{2}$ veto on the $\mathrm{H} 1$ and $\mathrm{H} 2$ data would have required at least $56.0 \mathrm{~h}$ and $7.0 \mathrm{~h}$ respectively. The price that is paid is that the search pipeline has a much more complicated topology. We will see in section 3 that even a small amount of data will yield a 


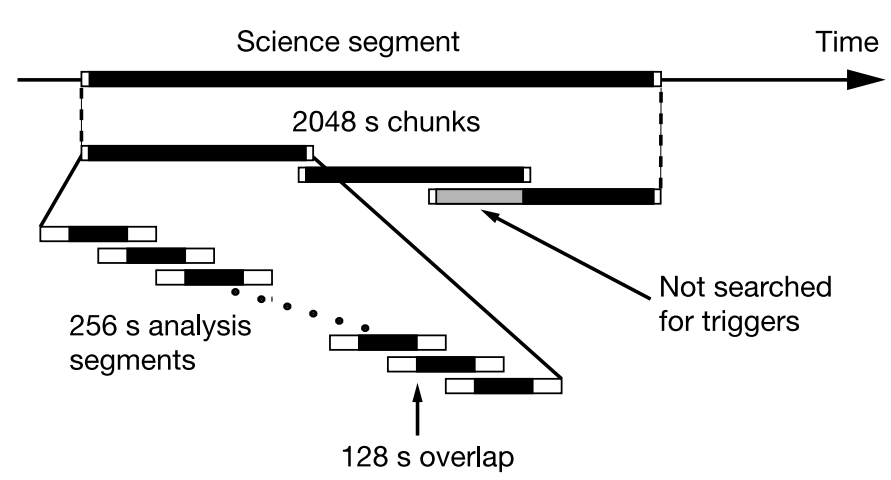

Figure 2. The algorithm used to divide science segments into data analysis segments. Science segments are divided into $2048 \mathrm{~s}$ chunks overlapped by $128 \mathrm{~s}$. (Science segments shorter than $2048 \mathrm{~s}$ are ignored.) An additional chunk with a larger overlap is added to cover any remaining data at the end of a science segment. Each chunk is divided into 15 analysis segments of length $256 \mathrm{~s}$ for filtering. The first and last $64 \mathrm{~s}$ of each analysis segment is ignored, so the segments overlap by $128 \mathrm{~s}$. Areas shaded black are filtered for triggers by the search pipeline. The grey area in the last chunk of the science segment is not searched for triggers as this time is covered by the preceding chunk; however, these data are used in the PSD estimate for the final chunk.

rather complex set of dependences between programs and their data products. First we will give a brief description of these programs.

\subsection{The DATAFIND program}

The DATAFIND program [8] uses tables of science segments-lists of time when each interferometer was operating stably and producing 'science mode' data-to identify data chunks to be used in the analysis. The chunks are composed of 15 overlapping analysis segments of $256 \mathrm{~s}$ which are used for power spectral density (PSD) estimation and for matched filtering with the FINDCHIRP algorithm. For the S2 inspiral analysis chunks of $2048 \mathrm{~s}$ are used and are chosen so that they overlap by $128 \mathrm{~s}$ to allow continuous filtering across the boundary between chunks (though the last chunk in a science segment overlaps by a larger amount so that it can remain $2048 \mathrm{~s}$ long). Figure 2 shows how analysis chunks are constructed from science segments in the S2 binary neutron star and binary black hole MACHO inspiral analyses.

\subsection{The TMPLTBANK program}

The тмРLтвANK program [9] is responsible for producing a bank of waveform templates to be used as matched filters by the INSPIRAL program. In the triggered search pipeline the L1 data are filtered first and since the coincidence stage requires triggers to be observed in the same template (see below), it is sufficient to generate one template bank that is suitable for the L1 data. To construct a template bank TMPLTBANK needs an estimate of the average strain noise PSD of the instrument. This is computed for each data analysis chunk (as provided by DATAFIND) using the same method as the INSPIRAL code. The specific templates that produce inspiral triggers in the INSPIRAL program will then be used as the bank for subsequent analyses of $\mathrm{H} 1$ and/or $\mathrm{H} 2$ data.

The S2 binary neutron star and binary black hole MACHO inspiral searches looked for signals from binary systems where the component masses were in the range $1 M_{\odot} \leqslant$ $m_{1}, m_{2} \leqslant 3 M_{\odot}$ (binary neutron stars) and the range $0.2 M_{\odot} \leqslant m_{1}, m_{2} \leqslant 1 M_{\odot}$ (binary black hole MACHOs). Since each mass pair $m_{1}, m_{2}$ in the space produces a different waveform, we construct a template bank-a discrete subset of the continuous family of waveforms that 
belong to the parameter space. The placement of the templates in the bank is determined by the mismatch of the bank which is the maximum fractional loss of signal-to-noise ratio that can occur by filtering a true signal with component masses $m_{1}, m_{2}$ with the 'nearest' template waveform for a system with component masses $m_{1}^{\prime}, m_{2}^{\prime}$. The construction of an appropriate template bank is discussed in $[10,11]$.

A typical choice for the maximum bank mismatch is $3 \%$, which would correspond to a $10 \%$ loss of event rate for a population of sources with uniform spatial distribution. This $3 \%$ bank mismatch was adopted in the S2 binary neutron star search. Binary black hole MACHO coalescences in the Milky Way halo would be easily detectable; it is not so important for the template bank to have such a small mismatch. By raising the mismatch, fewer templates are needed in the bank. This is an important computational-cost saving strategy for the binary black hole MACHO search as the template bank is very large for these very-low mass sources. For the S2 binary black hole MACHO search we adopted a mismatch of $5 \%$.

\subsection{The INSPIRAL program}

The INSPIRAL program [9] is the driver for the FINDCHIRP algorithm, which is discussed in detail in [6]. This program acquires a chunk of data identified by DATAFIND and the bank of templates produced by TMPLTBANK or TRIGBANK. The INSPIRAL program then (i) conditions the data by applying an eighth order Butterworth high-pass filter with $10 \%$ attenuation at 100 $\mathrm{Hz}$ and resamples the data from $16384 \mathrm{~Hz}$ to $4096 \mathrm{~Hz}$, (ii) acquires the current calibration information for the data, (iii) computes an average power spectrum from the 15 analysis segments, and (iv) filters the analysis segments against the bank of filter templates and applies the $\chi^{2}$ test for waveform consistency. These operations (apart from the data conditioning) are described in detail in the paper discussing the FINDCHIRP algorithm. The INSPIRAL program requires several threshold levels: the signal-to-noise threshold $\rho_{*}$, the chi-squared threshold $\chi_{*}^{2}$, the number of bins $p$ to use in the chi-squared veto, and the parameter $\delta$ used to account for the mismatch of a signal with a template when implementing the chi-squared veto. Several additional parameters used by the INSPIRAL program include the duration (and therefore the number) of analysis segments, the high-pass filter order and frequency, the low-frequency cutoff for the waveform templates and the inverse spectrum truncation duration $( \pm 16 \mathrm{~s})$.

The INSPIRAL program produces a list of inspiral triggers that have crossed the signal-tonoise ratio threshold and have survived the chi-squared veto. These triggers must then be confronted with triggers from other detectors to look for coincidences.

\subsection{The TRIGBANK program}

The TRIGBANK program [9] converts a list of triggers coming from INSPIRAL and constructs an appropriate template bank that is optimized to minimize the computational cost in a follow-up stage. Only those templates that were found in one detector need to be used as filters in the follow-up analysis, and only the data segments of the second detector where triggers were found in the data from the first detector need to be analysed. Thus, in the S2 triggered search pipeline, only the L1 data set needs to be fully analysed; only a small fraction of the $\mathrm{H} 1$ and $\mathrm{H} 2$ data sets needs to be analysed in the follow-up, and then only a fraction of the template bank needs to be used. The template bank produced by TRIGBANK is read by the INSPIRAL program that is run on the second detector's data.

\subsection{The INCA program}

The inspiral coincidence analysis, INCA [9], performs several tests for consistency between triggers produced by INSPIRAL output from analysing data from two detectors. Triggers are 
Table 1. The fake science segments used to construct the DAG shown in figure 3. Start and end time is given in GPS time (seconds since $0 \mathrm{~h}$ UTC 6 Jan 1980).

\begin{tabular}{lccc}
\hline Interferometer & Start time $(\mathrm{s})$ & End time $(\mathrm{s})$ & Duration $(\mathrm{s})$ \\
\hline L1 & 730000000 & 730010000 & 10000 \\
L1 & 731001000 & 731006000 & 5000 \\
L1 & 732000000 & 732003000 & 3000 \\
H1 & 730004000 & 730013000 & 8000 \\
H1 & 731000000 & 731002500 & 2500 \\
H1 & 732000000 & 732003000 & 3000 \\
H2 & 730002500 & 730008000 & 5500 \\
H2 & 731004500 & 731007500 & 2500 \\
H2 & 732000000 & 732003000 & 3000 \\
\hline
\end{tabular}

said to be coincident if they have consistent start times (taken to be $\pm 1 \mathrm{~ms}$ for coincidence between triggers from $\mathrm{H} 1$ and $\mathrm{H} 2$ and taken to be $\pm 11 \mathrm{~ms}$ for coincidence between Hanford and Livingston triggers, where $10 \mathrm{~ms}$ is the light travel time between the two sites and $1 \mathrm{~ms}$ is taken to be the timing accuracy of our matched filter). The triggers must also be in the same waveform template and the measured effective distance, $D_{\mathrm{H} 1}$ and $D_{\mathrm{H} 2}$ in $\mathrm{H} 1$ and $\mathrm{H} 2$ respectively, of the putative inspiral must agree in triggers from $\mathrm{H} 1$ and $\mathrm{H} 2:\left|D_{\mathrm{H} 1}-D_{\mathrm{H} 2}\right| / D_{\mathrm{H} 1}<\kappa+\epsilon / \rho_{\mathrm{H} 2}$ where $\kappa$ and $\epsilon$ are tunable parameters and $\rho_{\mathrm{H} 2}$ is the signal-to-noise ratio observed in $\mathrm{H} 2$. Such an amplitude consistency is not applied in the coincidence requirements between Hanford and Livingston triggers because the difference in detector alignment at the sites, while small, is significant enough that a significant fraction of possible signals could appear with large amplitude ratios. In addition, in comparing Hanford triggers, it is important not to discard $\mathrm{H} 1$ triggers that fail to be coincident with $\mathrm{H} 2$ triggers merely because $\mathrm{H} 2$ was not sensitive enough to detect that trigger. Therefore $\mathrm{H} 1 / \mathrm{H} 2$ coincidence is performed as follows. (1) For each $\mathrm{H} 1$ trigger compute $(1-\kappa) D_{\mathrm{H} 1} /\left(1+\epsilon / \hat{\rho}_{\mathrm{H} 2}\right)$ (the lower bound on the allowed range of effective distance) and $(1+\kappa) D_{\mathrm{H} 1} /\left(1-\epsilon / \hat{\rho}_{\mathrm{H} 2}\right)$ (the upper bound of the range). Here $\hat{\rho}_{\mathrm{H} 2}$ is the anticipated signal-to-noise ratio of a trigger in $\mathrm{H} 2$ given the observed trigger in $\mathrm{H} 1$. (2) Compute the maximum range of $\mathrm{H} 2$ for the trigger mass. (3) If the lower bound on the effective distance is further away than can be seen in $\mathrm{H} 2$, keep the trigger (do not require coincidence in $\mathrm{H} 2$ ). (4) If the range of $\mathrm{H} 2$ lies between the lower and upper bounds on the effective distance, keep the trigger in $\mathrm{H} 1$. If an $\mathrm{H} 2$ trigger is found within the interval, store it as well. (5) If the upper bound on the distance is less than the range of $\mathrm{H} 2$, check for coincidence. If there is no coincident trigger discard the $\mathrm{H} 1$ trigger.

\section{Construction of the search pipeline}

In this section, we demonstrate how the S2 triggered search pipeline in figure 1 can be abstracted into a DAG to execute the analysis. We illustrate the construction of the DAG with the short list of science segments shown in table 1 . For simplicity, we only describe the construction of the DAG for zero time lag results ${ }^{1}$. The DAG we construct filters more than the absolute minimum amount of data needed to cover all the double and triple coincident

\footnotetext{
1 An artificial delay in the trigger time can be introduced to produce false coincidences-i.e., coincidences that cannot arise from astrophysical signals - as a means to obtain information on the background event rate. We refer to this as non-zero time lag results.
} 
data, but since we were not computationally limited during S2, we chose simplicity over the maximum amount of optimization that we could have used.

A DAG consists of nodes and edges. The nodes are the programs which are executed to perform the inspiral search pipeline. In the S2 triggered search pipeline, the possible nodes of the DAG are DATAFInD, TMPLTBANK, InSPIRAL, TRIGBANK and INCA. The edges in the DAG define the relations between programs; these relations are determined in terms of parents and children, hence the directed nature of the DAG. A node in the DAG will not be executed until all of its parents have been successfully executed. There is no limit to the number of parents a node can have; it may be zero or many. In order for the DAG to be acyclic, no node can be a child of any node that depends on the execution of that node. By definition, there must be at least one node in the DAG with no parents. This node is executed first, followed by any other nodes who have no parents or whose parents have previously been executed. The construction of a DAG allows us to ensure that inspiral triggers for two interferometers have been generated before looking for coincidence between the triggers, for example.

The $\mathrm{S} 2$ inspiral DAG is generated by a script which is an implementation of the logic of the S2 triggered search pipeline. The script reads in all science segments longer than 2048 seconds and divides them into master analysis chunks. If there are data at the end of a science segment that are shorter than $2048 \mathrm{~s}$, the chunk is overlapped with the previous one, so that the chunk ends at the end of the science segment. An option is given to the INSPIRAL code that ensures that no triggers are generated before this time and so no triggers are duplicated between chunks. Although the script generates all the master chunks for a given interferometer, not all of them will necessarily be filtered. Only those that overlap with double or triple coincident data are used for analysis. The master analysis chunks are constructed for L1, H1 and H2 separately by reading in the three science segment files. For example, the first L1 science segment in table 1 is divided into the master chunks with GPS times 730000000-730002048, 730001920730003968,730003 840-730 005 888, 730 005 760-730 007 808, 730 007 680-730 009728 , and $730007952-730010000$ (with triggers starting at time 730009664 ).

The pipeline script next computes the disjoint regions of double and triple coincident data to be searched for triggers. $64 \mathrm{~s}$ are subtracted from the start and end of each science segment (since these data are not searched for triggers) and the script performs the correct intersection and unions of the science segments from each interferometer to generate the segments containing the times of science mode data to search. The L1/H1 double coincident segments are 730007 936-730 009936 and $731001064-731002436$; the L1/H2 double coincident segments are 730002 564-730004 064 and 731004 564-731005 936; and the $\mathrm{L} 1 / \mathrm{H} 1 / \mathrm{H} 2$ triple coincident segments are 730004064-730007936 and 732000064 732 002936 . The GPS start and end times are thus given for each segment to be searched for triggers. The script uses this list of science data to decide which master analysis chunks need to be filtered. All L1 master chunks that overlap with $\mathrm{H} 1$ or $\mathrm{H} 2$ science data to be searched are filtered. An L1 template bank is generated for each master chunk and the L1 data are filtered using this bank. This produces two intermediate data products for each master chunk, which are stored as XML data. The intermediate data products are the template bank file, L1-TMPLTBANK-730000000-2048.xml, and the inspiral trigger file, L1-INSPIRAL-730000000-2048.xml. The GPS time in the file name corresponds to the start time of the master chunk filtered and the number before the .xml file extension is the length of the master chunk.

All $\mathrm{H} 2$ master chunks that overlap with the $\mathrm{L} 1 / \mathrm{H} 2$ double coincident data to filter are then analysed. For each $\mathrm{H} 2$ master chunk, a triggered template bank is generated from L1 triggers between the start and end time of the $\mathrm{H} 2$ master chunk. The triggered bank file generated is 
called H2-TRIGBANK_L1-730002500-2048.xml, where the GPS time corresponds to the start time of the master $\mathrm{H} 2$ chunk to filter. All L1 master chunks that overlap with the $\mathrm{H} 2$ master chunk are used as input to the triggered bank generation to ensure that all necessary templates are filtered. The $\mathrm{H} 2$ master chunks are filtered using the triggered template bank for that master chunk to produce H2 triggers in files named H2-INSPIRAL_L1-730002500-2048.xml. The GPS start time in the file name is the start time of the $\mathrm{H} 2$ master chunk.

All H1 master chunks that overlap with either the $\mathrm{L} 1 / \mathrm{H} 1$ double coincident data or the $\mathrm{L} 1 / \mathrm{H} 1 / \mathrm{H} 2$ triple coincident data are filtered. The bank and trigger generation is similar to the $\mathrm{L} 1 / \mathrm{H} 2$ double coincident case. The triggered template bank is stored in a file named H1-TRIGBANK_L1-730004000-2048.xml and the triggers in a file named H1-INSPIRAL_L1-730004000-2048.xml where the GPS time in the file name is the GPS start time of the $\mathrm{H} 1$ master chunk. The $\mathrm{H} 2$ master chunks that overlap with the $\mathrm{L} 1 / \mathrm{H} 1 / \mathrm{H} 2$ triple coincident data are described below.

For each L1/H1 double coincident segment to search, an INCA process is run to perform the coincidence test. The input to INCA is all L1 and H1 master chunks that overlap the segment to search. The GPS start and stop times passed to INCA are the start and stop times of the double coincident segment to search. The output is a file named H1-INCA_L1H1-730007936-2000.xml. The GPS start time in the file name is the start time of the double coincident segment. A similar procedure is followed for each L1/H2 double coincident segment to search. The output files from INCA are named H2-INCA_L1H2-731004564-1372.xml, and so on.

For each $\mathrm{L} 1 / \mathrm{H} 1 / \mathrm{H} 2$ triple coincident segment, an INCA process is run to create the $\mathrm{L} 1 / \mathrm{H} 1$ coincident triggers for this segment. The input files are all L1 and H1 master chunks that overlap with the segment. The start and end times to INCA are the start and end times of the segment. This creates a file named H1-INCA_L1H1T-730004064-3872.xml where the GPS start time and duration in the file name are those of the triple coincident segment to search. For coincidence between L1 and a Hanford interferometer, we only check for time and mass coincidence (the amplitude cut is disabled).

For each $\mathrm{H} 2$ master chunk that overlaps with triple coincident data, a triggered template bank is generated. The input file to the triggered bank generation is the INCA file for the segment to filter that contains the master chunk. The start and end times of the triggered bank generation are the start and end times of the master chunk. This creates a file called H2-TRIGBANK_L1H1-730004420-2048.xml. The H2 master chunk is filtered through the INSPIRAL code to produce a trigger file H2-INSPIRAL_L1H1-730004420-2048.xml.

For each triple coincident segment to filter, an INCA is run between the H1 triggers from the L1H1T INCA and the $\mathrm{H} 2$ triggers produced by the inspiral code. The input files are the H1 INCA file H1-INCA_L1H1T-730004064-3872.xml and all H2 master chunk inspiral trigger files that overlap with this interval. An $\mathrm{H} 1 / \mathrm{H} 2$ coincidence step creates two files: H1-INCA_L1H1H2-730004064-3872.xml and H2-INCA_L1H1H2-730004064-3872.xml where the GPS start time and duration of the files are the start and duration of the triple coincident segment. The L1/H1 coincidence step is then executed again to discard any L1 triggers not coincident with the final list of $\mathrm{H} 1$ triggers. Input to the INCA are the files L1-INCA_L1H1T-730004064-3872.xml and H1-INCA_L1H1H2-730004064-3872.xml. Output are the files L1-INCA_L1H1H2-730004064-3872.xml and H1-INCA_L1H1H2730004064-3872.xml. The H1 input file is overwritten as it is identical to the H1 output file.

Finally, we obtain the data products of the search which contain the candidate trigger found by the S2 triggered search pipeline in these fake segments. For the fake segments described here, the final output files will be 


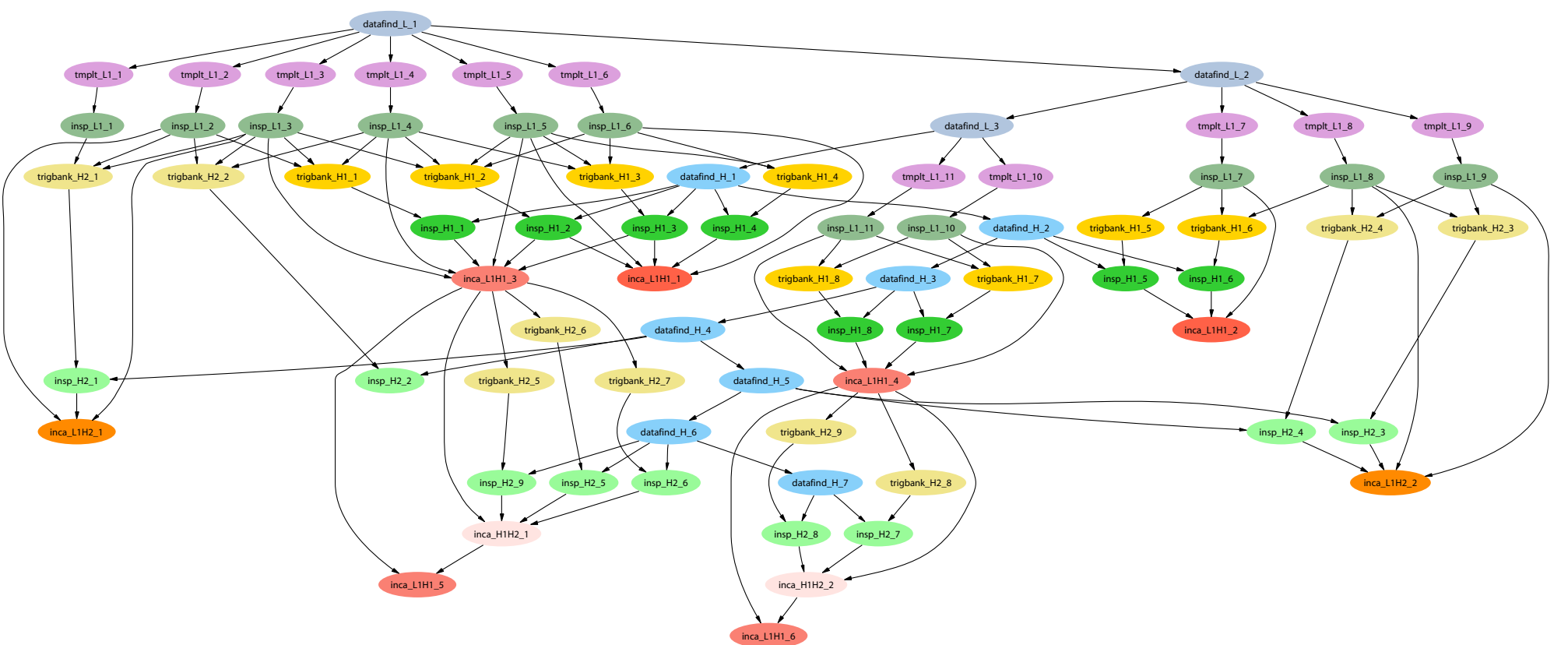

Figure 3. The DAG generated from the pipeline shown in figure 1 and the fake science segment list described in table 1. The figure shows the structure of the DAG with all job dependences needed to execute the S2 triggered search pipeline. Note that it appears that there are several Hanford master chunks analysed that do not need to be filtered for a zero time lag. These are added to the DAG to ensure that all the data necessary for a background estimation with a maximum time side of $500 \mathrm{~s}$ are analysed. 
Double coincident L1/H1 data

L1-INCA_L1H1-730007936-2000.xml L1-INCA_L1H1-731001064-1372.xm]

H1-INCA_L1H1-730007936-2000.xml H1-INCA_L1H1-731001064-1372.xml

Double coincident L1/H2 data

L1-INCA_L1H2-730002564-1500.xml L1-INCA_L1H2-731004564-1372.xml

H2-INCA_L1H2-730002564-1500.xml H2-INCA_L1H2-731004564-1372.xml

Triple coincident $\mathrm{LI} / \mathrm{H1} / \mathrm{H} 2 \mathrm{data}$

L1-INCA_L1H1H2-730004064-3872.xml L1-INCA_L1H1H2-732000064-2872.xml

H1-INCA_L1H1H2-730004064-3872.xml H1-INCA_L1H1H2-732000064-2872.xml

H2-INCA_L1H1H2-730004064-3872.xml H2-INCA_L1H1H2-732000064-2872.xml

The DAG that results from the fake science segments given in table 1 is shown graphically in figure 3. As the size of the input science segment files increases, so the number of nodes and vertices in the DAG increases; however, the algorithm for generating the DAG remains the same. While the example DAG shown in figure 3 has only 76 nodes, the analysis of the full S2 data set required a DAG containing 6941 nodes (for the zero-lag analysis alone). The results of the execution of this DAG to analyse the $S 2$ data will be reported in $[3,4]$.

Although the use of the triggered search pipeline greatly alleviates the computational burden required in an analysis, manually shepherding data through this complicated pipeline would clearly be prohibitive; even very simple pipelines benefit greatly from the use of DAGs (whose creation can be automated) to describe the organization of tasks and dataflow and a batch processing environment such as Condor to execute the DAG in a fault-tolerant manner.

\section{Acknowledgments}

The authors gratefully acknowledge the support of the United States National Science Foundation for the construction and operation of the LIGO Laboratory and the Particle Physics and Astronomy Research Council of the United Kingdom, the Max-Planck-Society and the State of Niedersachsen/Germany for support of the construction and operation of the GEO600 detector. The authors also gratefully acknowledge the support of the research by these agencies and by the Australian Research Council, the Natural Sciences and Engineering Research Council of Canada, the Council of Scientific and Industrial Research of India, the Department of Science and Technology of India, the Spanish Ministerio de Educacion y Ciencia, the John Simon Guggenheim Foundation, the Leverhulme Trust, the David and Lucile Packard Foundation, the Research Corporation, and the Alfred P Sloan Foundation.

\section{References}

[1] Abbott B et al 2004 Phys. Rev. D 69122001

[2] Messaritaki E (for the LIGO Scientific Collaboration) 2005 Report on the first binary black hole inspiral search on LIGO data Class. Quantum Grav. 22 S1119

Abbott B et al LIGO search for gravitational waves from binary black hole mergers, in preparation

[3] Abbott B et al 2005 Search for gravitational waves from galactic and extra-galactic binary neutron stars Preprint gr-qc/0505041 (Phys. Rev. D submitted)

[4] Abbott B et al 2005 Search for gravitational waves from primordial black hole binary coalescences in the Galactic Halo Phys. Rev. D at press (Preprint gr-qc/0505042)

[5] Brown D A 2004 Searching for gravitational radiation from black hole MACHOs in the Galactic halo PhD Thesis University of Wisconsin-Milwaukee

[6] Allen B et al FINDCHIRP: an algorithm for the detection of gravitational waves from inspiraling compact binaries, in preparation 
[7] Condor High Throughput Computing System http://www.cs.wisc.edu/condor

[8] The DATAFIND program is part of the Lightweight Data Replicator (LDR) http://www.lsc-group.phys.uwm.edu/ LDR

[9] The programs TMPLTBANK, INSPIRAL, TRIGBANK, and INCA are in the LSC Algorithm Library Applications software package LALAPPS: http://www.lsc-group.phys.uwm.edu/daswg/projects/lalapps.html

[10] Owen B J 1996 Phys. Rev. D 536749

[11] Owen B J and Sathyaprakash B S 1999 Phys. Rev. D 60022002 